\title{
Risk Factors for Incisional and Organ Space Surgical Site Infections After Liver Resection are Different
}

\author{
Takashi Kokudo $\cdot$ Emilie Uldry $\cdot$ Nicolas Demartines $•$ \\ Nermin Halkic
}

Published online: 6 January 2015

(C) Société Internationale de Chirurgie 2014

\begin{abstract}
Background Surgical site infection (SSI) is a common cause of major morbidity after liver resection. This study aimed to identify the risk factors for incisional and organ/space SSIs after liver resection.

Methods Our liver surgery database was retrospectively analyzed for patients treated between January 2009 and November 2012 in a tertiary care Swiss hospital. Univariate and multivariate analyses were conducted on preoperative, intraoperative, and postoperative variables to identify risk factors for incisional and organ/space SSIs.

Results In a total of 226 patients, SSI incidences were $12.8 \%$ (incisional), $4.0 \%$ (organ/space), and $1.8 \%$ (both). Univariate analysis showed that incisional SSIs were associated with high American Society of Anesthesiologists (ASA) scores, preoperative anemia, hypoalbuminemia, low prothrombin time, viral or alcoholic chronic hepatitis, liver cirrhosis, and prolonged operation times. Organ/space SSIs were associated with high rates of red blood cell transfusions, concomitant bowel surgery, and prolonged operation times. Multivariate analysis revealed that risk factors for incisional SSIs were anemia [odds ratio (OR) 2.82], high ASA scores (OR 2.88), presence of hepatitis or cirrhosis (OR 5.07), and prolonged operation times (OR 9.61). The only risk factor for organ/space SSIs was concomitant bowel surgery (OR 5.53). Hospital stays were similar in organ/space and incisional SSI groups, but significantly longer for those with both organ/space and incisional SSIs.

Conclusions High ASA scores, anemia, chronic hepatitis or liver cirrhosis, and prolonged operations increased the risk of incisional SSIs; concomitant bowel surgery increased the risk of organ/space SSI. Specific precautions to prevent organ/space and incisional SSIs may shorten hospital stays.
\end{abstract}

\section{Introduction}

As a result of recent advances in surgical technology and perioperative management, liver resection has become a safe surgery with a low mortality rate [1, 2]. However, morbidity rates remain relatively high. Bile leakage and surgical site infection (SSI) are common causes of major

T. Kokudo $\cdot$ E. Uldry $\cdot$ N. Demartines $(\bowtie) \cdot$ N. Halkic

Department of Visceral Surgery, University Hospital CHUV,

Rue du Bugnon 46, 1011 Lausanne, Switzerland

e-mail: Demartines@chuv.ch

T. Kokudo

e-mail: kokudo-tky@umin.ac.jp morbidity after liver resection [3-5]. The incidence of SSI after hepatectomy has been reported to be 3.1-14\% [4-8]. The risk factors for SSI have been widely studied for colorectal surgery; significant risk factors include overweight status, high American Society of Anesthesiologists (ASA) score, and long operation times [9-11]. Ho et al. [11] recently reported that different risk factors and clinical outcomes were associated with incisional SSIs and organ and/or space SSIs; thus, a specific approach was required for each type of SSI. In liver surgery, risk factors associated with all SSIs have been reported, but not separate risk factors associated with either incisional or organ/space SSIs [12]. 
Recently, specific analyses of both incisional and organ/ space SSIs after liver resection for hepatocellular carcinoma were conducted in Japanese patients with cirrhosis $[3,13,14]$. In the present study, we evaluated clinical risk factors associated with either incisional or organ/space SSIs after elective liver resections in a European population where colorectal liver metastasis was the leading indication for liver resection.

\section{Materials and methods}

All consecutive patients scheduled for elective liver resection from January 2009 to November 2012 were included in this study. Demographic, clinical, and radiological data were recorded prospectively and analyzed retrospectively. The extent of hepatectomy was evaluated according to stage of the disease, liver function, and the general condition of patients. Stage of the disease and resectability was assessed with imaging studies, including contrast-enhanced computed tomography, magnetic resonance imaging, hepatic arterial angiography, and ultrasound. The extent of liver function impairment due to underlying liver disease was assessed with a liver biochemistry test, the Child-Pugh grade, and the indocyanine green retention rate at 15 min [15].

The investigated independent risk factors included preoperative, intraoperative, and postoperative variables (Tables 1 and 2). Body mass indexes (BMIs) greater than 25 and $30 \mathrm{~kg} / \mathrm{m}^{2}$ were defined as overweight status and obesity, respectively [16]. The ASA score was divided into two groups of ASA $=1$ or 2 and ASA $=3$. No patient had an $\mathrm{ASA}=4$ in the study group.
Table 1 Preoperative characteristics of patients that did or did not develop incisional SSIs
ASA American society of anesthesiologists, $H B V$ hepatitis $\mathrm{B}$ virus, $H C V$ hepatitis $\mathrm{C}$ virus

${ }^{\text {a }}$ Bile duct cancer, intrahepatic cholangiocarcinoma, gallbladder cancer, and metastases from cancers other than colorectal cancer

\begin{tabular}{|c|c|c|c|}
\hline & Incisional SSIs $n=29(\%)$ & No SSI $n=197(\%)$ & $p$ values \\
\hline \multicolumn{4}{|l|}{ Age (years) } \\
\hline$\geq 65$ & $14(48)$ & $95(48)$ & \multirow[t]{2}{*}{1.00} \\
\hline$<65$ & $15(52)$ & $102(52)$ & \\
\hline Gender (male/female) & $21 / 8(72 / 28)$ & $126 / 71(64 / 36)$ & 0.37 \\
\hline \multicolumn{4}{|l|}{ ASA score } \\
\hline $1-2$ & $15(52)$ & $151(77)$ & \multirow[t]{2}{*}{0.005} \\
\hline 3 & $14(48)$ & $46(23)$ & \\
\hline \multicolumn{4}{|l|}{ Body mass index $\left(\mathrm{kg} / \mathrm{m}^{2}\right)$} \\
\hline$<25$ & $13(45)$ & $101(51)$ & \multirow[t]{2}{*}{0.52} \\
\hline$\geq 25$ & $16(55)$ & $96(49)$ & \\
\hline Diabetes mellitus & $8(28)$ & $29(15)$ & 0.08 \\
\hline \multicolumn{4}{|l|}{ Laboratory data } \\
\hline Hemoglobin $<12.0 \mathrm{~g} / \mathrm{dl}$ & $13(45)$ & $50(25)$ & $\mathrm{P}=0.03$ \\
\hline Albumin $<3.5 \mathrm{~g} / \mathrm{dl}$ & $6(21)$ & $15(8)$ & $\mathrm{P}=0.02$ \\
\hline Prothrombin time $<80 \%$ & $10(35)$ & $24(12)$ & 0.002 \\
\hline \multicolumn{4}{|l|}{ Underlying liver diseases } \\
\hline None & $15(52)$ & $163(83)$ & 0.02 \\
\hline Chronic hepatitis & $6(21)$ & $14(7)$ & \multirow[t]{2}{*}{0.008} \\
\hline Liver cirrhosis & $8(28)$ & $20(10)$ & \\
\hline \multicolumn{4}{|l|}{ Etiology of hepatic diseases } \\
\hline $\mathrm{HBV}$ and/or HCV & $5(17)$ & $11(6)$ & 0.04 \\
\hline Alcoholic & $7(24)$ & $13(7)$ & 0.002 \\
\hline Unknown & $2(7)$ & $10(5)$ & 0.66 \\
\hline Preoperative chemotherapy & $13(45)$ & $90(46)$ & 0.93 \\
\hline Primary or repeat hepatectomy & $25 / 4(86 / 14)$ & $164 / 33(83 / 17)$ & 1.00 \\
\hline Tobacco use & $13(45)$ & $91(46)$ & 0.89 \\
\hline \multicolumn{4}{|l|}{ Indications for surgery } \\
\hline Hepatocellular carcinoma & $8(28)$ & $30(15)$ & 0.10 \\
\hline Colorectal metastasis & $10(34)$ & $100(51)$ & 0.10 \\
\hline Other malignancy ${ }^{\mathrm{a}}$ & $6(21)$ & $36(18)$ & 0.76 \\
\hline Echinococcus alveolar & $2(7)$ & $13(7)$ & 1.00 \\
\hline Benign and pseudo tumor & $3(10)$ & $18(9)$ & 0.74 \\
\hline
\end{tabular}


Table 2 Intraoperative and postoperative characteristics of patients that did or did not develop incisional SSIs
$R B C$ red blood cells, $F F P$ fresh frozen plasma

a More than 3 Couinaud's segments

b Classified higher than Grade II in Dindo-Clavien classification [21]

\begin{tabular}{lccc}
\hline & Incisional SSIs $n=29(\%)$ & No SSI $n=197(\%)$ & $p$ values \\
\hline Intraoperative variables & & & \\
Median operative time; min (IQR) & $375(335-414)$ & $283(269-298)$ & 0.001 \\
Major hepatic resection $^{\text {a }}$ & $16(55)$ & $74(38)$ & 0.07 \\
Blood loss $>500 \mathrm{ml}$ & $19(66)$ & $94(48)$ & 0.07 \\
Pringle's maneuver & $20(69)$ & $137(70)$ & 0.95 \\
Concomitant bowel surgery & $6(21)$ & $19(10)$ & 0.08 \\
Minimally invasive approach & $1(3)$ & $15(8)$ & 0.70 \\
Required RBC transfusion & $12(41)$ & $52(26)$ & 0.10 \\
Required FFP transfusion & $4(14)$ & $13(7)$ & 0.25 \\
Postoperative variables & & & \\
Complications & & $20(10)$ & 0.001 \\
Bile leakage & $11(38)$ & $29(15)$ & 0.001 \\
$\quad$ Ascites & $16(55)$ & $4(11-14)$ & 0.001 \\
Median hospital stay; days (IQR) & $27(20-34)$ & & 0.50 \\
90-day mortality rate & $1(3)$ & & \\
\hline
\end{tabular}

A SSI was defined as a condition where purulent discharge was observed, either in the skin or subcutaneous tissue (incisional) or in an organ or space that was manipulated during liver resection. It had to be observed within 30 days after the operation, with or without microbiological evidence, as described in the Center for Disease Control guidelines [17, 18]. Incisional and organ/ space SSIs were diagnosed by the surgeon that performed the postoperative follow-up. All patients were followed-up by the surgeon for 2-4 weeks after discharge and regularly thereafter, according to the underlying disease, which warranted a follow-up period of 30 days or longer.

Prophylactic antibiotics were given to all patients just before the skin incision and every $3 \mathrm{~h}$ during the operation. No antibiotic was given after the liver was resected [19]. Before the procedure, the skin of the abdomen was prepared by applying a povidone-iodine solution or chlorhexidine, according to our institutional recommendations. Hepatic resection was performed under intraoperative, ultrasonographic guidance, with or without inflow occlusion (Pringle's maneuver). During parenchymal resection, all blood vessels and bile ducts were ligated, whenever possible, with an absorbable multifilament (Polyglactin 910; Vicryl ${ }^{\circledR}$ ) or with a vessel clip. After the resection, the fascia was closed with continuous absorbable monofilament (Polydioxanone; $\operatorname{PDS}^{\circledR}$ ), the surgical incision was irrigated with saline solution, and the wound was closed with a nylon monofilament with interrupted, transdermal, and vertical mattress sutures. Skin sutures were removed between postoperative days 14 and 21, depending on the surgeon's professional opinion. Prophylactic, routine abdominal drainage was not performed [20].

Concomitant bowel surgery was defined as opening any digestive structure during the operation, including a small bowel resection, a colorectal resection, and a biliodigestive anastomosis. Minimally invasive liver resections were either purely laparoscopic or hand-assisted laparoscopic liver resections. Prolonged surgery was arbitrarily defined as a surgical time longer than $300 \mathrm{~min}$.

Postoperative abdominal complications were recorded and graded according to the Dindo-Clavien classification [21]. Complications were included in the analysis when they involved bile leakage and ascites classified higher than Grade II. The most frequent management for bile leakage was radiological intervention. Postoperative mortality was defined as any death during postoperative hospitalization or within 90 days after liver resection.

Statistical analyses were performed with Stata Release 12 software (STATA Corp, College Station, TX). Categorical variables were analyzed with the $\chi^{2}$ test or Fisher exact test, as appropriate. Concerning continuous variables, the influence on hospital stay was analyzed using the student's t test, and that of the operation time was analyzed using the MannWhitney $U$ test. The Bonferroni correction was used for multiple comparisons. $P$ values less than 0.05 were considered significant. All statistical analyses were two tailed and based on the intention-to-treat concept. Risk factors included in multivariate analyses were preoperative and intraoperative variables with $\mathrm{p}$ values less than 0.10 in the univariate analysis. Since the aim of this study was to identify independent predictors of SSIs for preventing them, postoperative variables - such as bile leakage and ascites-were excluded from the multivariate analysis. Albumin levels, prothrombin time, and hepatic disease etiology were highly correlated with underlying liver diseases (chronic hepatitis and liver cirrhosis); therefore, they were excluded from the multivariate analyses in the incisional SSI group. A binary logistic regression model was used to identify independent predictors of incisional and organ/space SSIs after liver resection. 


\section{Results}

During the study period, a total of 226 patients underwent liver resection. During the postoperative course, $12.8 \%$ of patients $(n=29)$ and $4.0 \%$ of patients $(n=9)$ were diagnosed with incisional and organ/space SSIs, respectively; $1.8 \%$ of patients $(n=4)$ were diagnosed with both. Patients with organ/space SSIs had significantly higher incidence of severe SSIs, classified higher than Grade III according to the Dindo-Clavien classification [21], than in those with incisional SSIs [8/9 (89\%) vs. 3/25 (12\%), $p<0.001]$. Five patients died after liver resection, and the cause of death was liver failure in 3 patients, suicide probably due to hepatic encephalopathy in 1 patient, and aspiration pneumonia in 1 patient. There was no case of SSI-related death.

Compared to those without SSIs, patients with incisional SSIs (Table 1) showed significantly higher frequencies of high ASA scores (ASA $=3,48$ vs. $23 \%, p=0.005$ ), preoperative anemia (hemoglobin $<12.0 \mathrm{~g} / \mathrm{dl}, 45$ vs. $25 \%$, $p=0.03$ ), hypoalbuminemia (albumin $<3.5 \mathrm{~g} / \mathrm{dl}, 21$ vs. $8 \%, p=0.02)$, low prothrombin times $(<80 \%, 35$ vs. $12 \%, p=0.002$ ), viral or alcoholic chronic hepatitis (21 vs. $7 \%, p=0.02$ ), and liver cirrhosis (28 vs. $10 \%, p=0.008$ ).

The intraoperative characteristics were similar between these groups (Table 2), except the median operation time, which was statistically longer in the incisional SSI than in the non-SSI group [375 min, interquartile range (IQR) 335-414 vs. 283 min, IQR 269-298; $p<0.001]$. Postoperatively, abdominal complications were significantly more frequent in the incisional SSI than in the non-SSI group (bile leakage, 38 vs. $10 \%, p<0.001$, and ascites 55 vs. $15 \%, p<0.001)$.

Multivariate analyses (Table 3 ) showed that preoperative anemia [odds ratio (OR) 2.82], a high ASA score (OR 2.88), the presence of chronic hepatitis or cirrhosis (OR 5.07), and a prolonged operation time (OR 9.61) were independently associated with the risk of incisional SSI after liver resection.

We also analyzed the risk factors in patients without liver cirrhosis. In this patient subgroup, patients with incisional SSIs showed significantly higher frequencies of high ASA scores (48 vs. $19 \%, p=0.006$ ), hypoalbuminemia (24 vs. $8 \%$, $p=0.04$ ), chronic hepatitis ( 29 vs. $8 \%, p=0.01$ ), intraoperative blood loss $>500 \mathrm{ml}$ (76 vs. $47 \%, p=0.02$ ), and longer operation time (370 min, IQR $334-436$ vs. $279 \mathrm{~min}$, IQR 222-340; $p<0.001)$. Multivariate analyses in the noncirrhotic patients group revealed that a high ASA score [OR 5.47, $95 \%$ confidence interval $(\mathrm{CI}) 1.76-18.06 ; p=0.003$ ], presence of chronic hepatitis (OR 5.70, $95 \%$ CI 1.32-24.9; $p=0.02$ ), concomitant bowel surgery (OR 4.29, $95 \% \mathrm{CI}$ $1.08-16.5 ; p=0.04)$, and a prolonged operation time (OR 15.0 , $95 \%$ CI $3.46-109$; $\mathrm{p}<0.001$ ) were independently associated with the risk of incisional SSI after liver resection.

Preoperative characteristics were similar for patients with or without organ/space SSIs after liver resection (Table 4).
Table 3 Risk factors for incisional or organ/space SSIs in multivariate analysis

\begin{tabular}{lcc}
\hline & $p$ value & Odds ratio $(95 \% \mathrm{CI})$ \\
\hline Incisional SSI & & \\
ASA score $=3$ & 0.03 & $2.88(1.09-7.61)$ \\
Diabetes mellitus & 0.69 & $1.25(0.42-3.78)$ \\
Hemoglobin $<12.0 \mathrm{~g} / \mathrm{dl}$ & 0.04 & $2.82(1.04-7.61)$ \\
Underlying liver diseases $^{\mathrm{a}}$ & 0.002 & $5.07(1.80-14.2)$ \\
Prolonged operation time $^{\mathrm{b}}$ & $<0.001$ & $9.61(2.87-32.1)$ \\
Major hepatic resection & 0.58 & $1.32(0.50-3.50)$ \\
Blood loss $>500 \mathrm{ml}$ & 0.36 & $0.62(0.22-1.71)$ \\
Concomitant bowel surgery & 0.20 & $2.30(0.65-8.21)$ \\
Organ/space SSI & & \\
Required RBC transfusion & 0.09 & $3.80(0.83-17.3)$ \\
Prolonged operation time & \\
Concomitant bowel surgery & 0.36 & $2.25(0.40-12.7)$ \\
\hline ASA American society of anesthesiologists, $R B C$ red blood cells \\
a Chronic hepatitis and/or liver cirrhosis \\
b Surgical time longer than 300 min
\end{tabular}

Intraoperative characteristics (Table 5) showed that patients with organ/space SSIs had significantly higher frequencies of red blood cell (RBC) transfusions (67 vs. $27 \%, p=0.02$ ), concomitant bowel surgery (44 vs. $10 \%, p=0.01$ ), and prolonged operation times (378 min, IQR 300-456 vs. 292 min, IQR 278-306; $p=0.02$ ). Postoperatively, bile leakage was significantly more frequent in the group with organ/space SSIs than in those without SSIs (67 vs. $12 \%$, $p<0.001)$.

Multivariate analyses (Table 3) showed that concomitant bowel surgery (OR 5.53) was an independent risk factor for organ/space SSI development after liver resection.

Compared to those with no SSIs, the 90-day mortality rates were not significantly different in patients with incisional SSIs ( 3 vs. $2 \%, p=0.50$ ) or those with organ/specific SSIs (0 vs. $2 \%, p=1.00)$. However, the hospital stay was significantly shorter in those without SSIs compared to the incisional SSI group (13 days, IQR 11-14 vs. 27 days, IQR 20-34; $p<0.001)$ and the organ/space SSI group (13 days, IQR $12-15$ vs. 38 days, IQR 16-60; $p<0.001)$. Furthermore, patients that developed both incisional and organ/space SSIs required much longer hospital stays (57 days, IQR 8-107) than either the incisional SSI group (22 days, IQR 18-26) or the organ/space SSI group (22 days, IQR 4-41) (Fig. 1; $p<0.001$ in multiple comparisons).

\section{Discussion}

The current study revealed that anemia, a high ASA score, the presence of hepatitis or cirrhosis, and a prolonged operation time were statistically significant risk factors for 
Table 4 Preoperative characteristics of patients that did or did not develop organ/ space SSIs

ASA American society of anesthesiologists, $H B V$ hepatitis $\mathrm{B}$ virus, $H C V$ hepatitis $\mathrm{C}$ virus

${ }^{\text {a }}$ Bile duct cancer, intrahepatic cholangiocarcinoma, gallbladder cancer, and metastases from cancers other than colorectal cancer

Table 5 Intraoperative and postoperative characteristics of patients that did or did not develop organ/space SSIs

$R B C$ red blood cells, FFP fresh frozen plasma

a More than 3 Couinaud's segments

b Classified higher than Grade II in Dindo-Clavien classification [21]

\begin{tabular}{|c|c|c|c|}
\hline & Organ/space SSIs $n=9(\%)$ & No SSI $n=217(\%)$ & $p$ values \\
\hline \multicolumn{4}{|l|}{ Age (years) } \\
\hline$\geq 65$ & $5(56)$ & $104(48)$ & \multirow[t]{2}{*}{0.74} \\
\hline$<65$ & $4(44)$ & $113(52)$ & \\
\hline Gender (male/female) & $5 / 4(56 / 44)$ & $142 / 75(65 / 35)$ & 0.72 \\
\hline \multicolumn{4}{|l|}{ ASA score } \\
\hline $1-2$ & $7(78)$ & $159(73)$ & \multirow[t]{2}{*}{1.00} \\
\hline 3 & $2(22)$ & $58(27)$ & \\
\hline \multicolumn{4}{|l|}{ Body mass index $\left(\mathrm{kg} / \mathrm{m}^{2}\right)$} \\
\hline$<25$ & $5(56)$ & $109(50)$ & \multirow[t]{2}{*}{1.00} \\
\hline$\geq 25$ & $4(44)$ & $108(50)$ & \\
\hline Diabetes mellitus & $2(22)$ & $35(16)$ & 0.64 \\
\hline \multicolumn{4}{|l|}{ Laboratory data } \\
\hline Hemoglobin $<12.0 \mathrm{~g} / \mathrm{dl}$ & $5(56)$ & $58(27)$ & 0.12 \\
\hline Albumin $<3.5 \mathrm{~g} / \mathrm{dl}$ & $2(22)$ & $19(9)$ & 0.20 \\
\hline Prothrombin time $<80 \%$ & $1(11)$ & $33(15)$ & 1.00 \\
\hline \multicolumn{4}{|l|}{ Underlying liver diseases } \\
\hline None & $8(89)$ & $170(78)$ & 1.00 \\
\hline Chronic hepatitis & $0(0)$ & $20(9)$ & \multirow[t]{2}{*}{1.00} \\
\hline Liver cirrhosis & $1(11)$ & $27(12)$ & \\
\hline \multicolumn{4}{|l|}{ Etiology of hepatic diseases } \\
\hline $\mathrm{HBV}$ and/or $\mathrm{HCV}$ & $1(11)$ & $15(7)$ & 0.49 \\
\hline Alcoholic & $0(0)$ & $20(9)$ & 1.00 \\
\hline Unknown & $0(0)$ & $12(6)$ & 1.00 \\
\hline Preoperative chemotherapy & $5(56)$ & $98(45)$ & 0.74 \\
\hline Primary or repeat hepatectomy & $6 / 3(67 / 33)$ & $183 / 34(84 / 16)$ & 0.17 \\
\hline Tobacco use & $3(33)$ & $101(47)$ & 0.51 \\
\hline \multicolumn{4}{|l|}{ Indications for surgery } \\
\hline Hepatocellular carcinoma & $1(11)$ & 37 (17) & 1.00 \\
\hline Colorectal metastasis & $5(56)$ & $105(48)$ & 0.74 \\
\hline Other malignancy $^{\mathrm{a}}$ & $2(22)$ & $40(18)$ & 0.68 \\
\hline Echinococcus alveolar & $0(0)$ & $15(7)$ & 1.00 \\
\hline \multirow[t]{2}{*}{ Benign and pseudo tumor } & $1(11)$ & $20(9)$ & 0.59 \\
\hline & Organ/space SSIs $n=9(\%)$ & No SSI $n=217(\%)$ & $p$ values \\
\hline \multicolumn{4}{|l|}{ Intraoperative variables } \\
\hline Median operative time; $\min$ (IQR) & $378(300-456)$ & $292(278-306)$ & 0.02 \\
\hline Major hepatic resection $^{\mathrm{a}}$ & $4(44)$ & $86(40)$ & 0.74 \\
\hline Blood loss $>500 \mathrm{ml}$ & $6(67)$ & $107(49)$ & 0.50 \\
\hline Pringle's maneuver & $4(44)$ & $153(71)$ & 0.14 \\
\hline Concomitant bowel surgery & $4(44)$ & $21(10)$ & 0.01 \\
\hline Minimally invasive approach & $0(0)$ & $16(7)$ & 1.00 \\
\hline Required RBC transfusion & $6(67)$ & $58(27)$ & 0.02 \\
\hline Required FFP transfusion & $2(22)$ & $15(7)$ & 0.14 \\
\hline \multicolumn{4}{|l|}{ Postoperative variables } \\
\hline \multicolumn{4}{|l|}{ Complications $^{\mathrm{b}}$} \\
\hline Bile leakage & $6(67)$ & $25(12)$ & 0.001 \\
\hline Ascites & $2(22)$ & $43(20)$ & 1.00 \\
\hline Median hospital stay; days (IQR) & $38(16-60)$ & $13(12-15)$ & 0.001 \\
\hline 90-day mortality rate & $0(0)$ & $5(2)$ & 1.00 \\
\hline
\end{tabular}




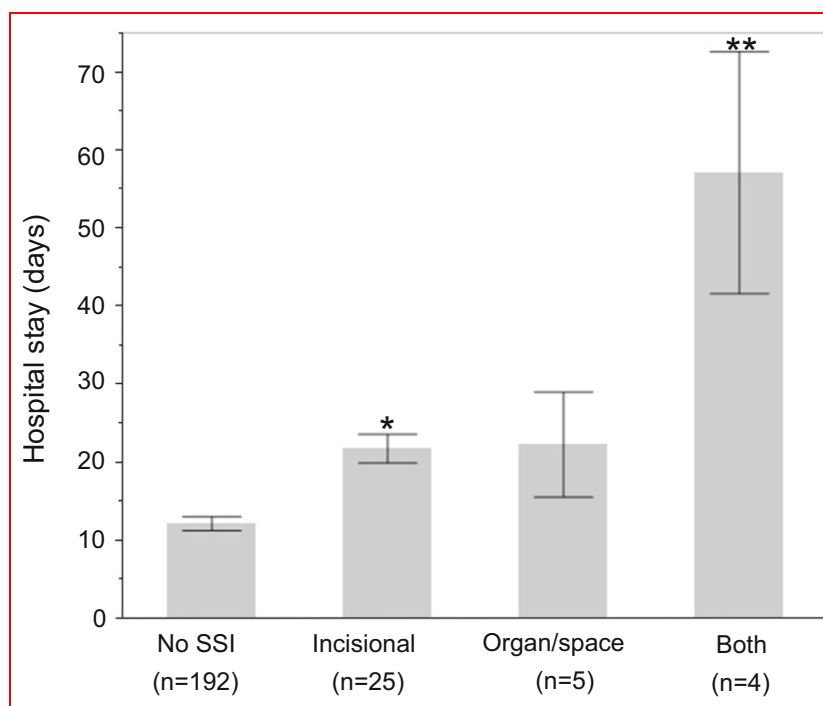

Fig. 1 Hospital stay according to types of SSIs. The error bars show the standard errors of the mean. Asterisk denotes statistically significant difference compared to the no-SSI group. Double asterisk denotes statistically significant difference compared to the no-SSI, incisional SSI, and organ/space SSI groups

incisional SSIs, and that only concomitant bowel surgery was a risk factor for organ/space SSIs. Of note, the risk factors were mainly preoperative factors related to compromise patient condition for incisional SSIs.

This study confirmed that there were different risk factors for incisional and organ/space SSIs; this was previously established for colorectal cancer [11]. Recently, large number of patients who underwent colectomy was analyzed and found that risk factors for incisional SSIs and organ/space SSIs vary in terms of magnitude and significance [22]. Findings from a recent Japanese study that also analyzed incisional and organ/space SSIs after liver resection supported this conclusion, although that study pointed to different risk factors than those identified in our study for a European population [3, 13]. Generally, incisional SSIs occur more frequently, but are less serious than organ/space SSIs, which are associated with a higher mortality [23]. Indeed, patients with organ/space SSIs had significantly higher incidence of severe SSIs than in those with incisional SSIs in the present study. The risk for developing any SSI is more affected by factors associated with incisional SSIs than with those associated with organ/ space SSIs, due to the different frequencies; however, this may be misleading when devising a strategy for preventing organ/space SSIs, which are clinically more important. Thus, it is important to evaluate risk factors separately for incisional and organ/space SSIs; different approaches may be necessary for prevention.

A review of the literature on risk factors for SSIs after liver resection is shown in Table 6 [3, 7, 8, 12, 13, 24-29]. The risk factors identified for incisional SSI in the present study were different from those identified in the Japanese study $[13,24]$. In the present study, BMI was not a significant risk factor, even in the univariate analysis. This was probably due to demographic differences between European and Asian populations, particularly in the prevalence of overweight status and obesity [30]. On the other hand, underlying liver disease was a significant risk factor in our study. This was probably due to the different frequencies of underlying liver disease between European and Japanese populations (21 vs. $77 \%)$ [13].

The risk factors identified for organ/space SSI in the present study were also different from those identified in the Japanese study $[3,26]$. The only risk factor for organ/ space SSIs in our study was concomitant bowel surgery. This could be due to different indications for surgery. In the Japanese study, liver resection was mainly performed for treating hepatocellular carcinoma; but this disease was rare in our study. In our study, liver resections were performed for treating colorectal metastases, and this more frequently required concomitant bowel surgery, including operations for removing a primary tumor.

Concomitant bowel surgery was the only identified risk factor for organ/space SSIs in our study. Although routine drainage is not recommended after a liver resection [20], the liver resection with concomitant bowel surgery may be an indication for drainage to prevent organ/space SSIs. This result was consistent with the previous finding that infectious complications increased with simultaneous hepatic and colorectal resections [31]. Avoiding simultaneous hepatic and colorectal resections would certainly be effective in preventing organ/space SSIs, but this option should be balanced with the risk of tumor progression in the interval between separate operations. Our data suggested that patients with both incisional and organ/space SSIs had significantly longer hospital stays than those with only one type of SSI. This indicated that it would be beneficial to avoid simultaneous hepatic and colorectal resections in patients with a high risk for incisional SSIs.

The clinical relevance of our study is that incisional SSIs and organ/space SSIs differ in their clinical impact, necessitating different prevention strategies for the two types of SSIs. Although there were no SSI-related mortalities in our study, organ/space SSIs were significantly more severe in terms of the Dindo-Clavien grade [21]. Furthermore, patients developing both incisional SSIs and organ/space SSIs required the longest hospital stay. For concurrent presence of both types of SSIs, concomitant bowel and liver surgery was the most important risk factor in patients without liver cirrhosis, especially in those with colorectal liver metastasis. This indicates that in compromised patients, e.g., those with high ASA scores, and with underlying chronic hepatitis or multiple liver metastases requiring a complicated surgical procedure with a long 
Table 6 List of previous publications on risk factors for SSIs after liver resection

\begin{tabular}{lll}
\hline Study no of subjects & Type of SSI & Risk factors \\
\hline Yoshimura [24] $n=296$ & Incisional & BMI, smoking, lack of drape use \\
Togo [25] $n=313$ & Both & Operative duration, silk sutures, bile leakage \\
Kobayashi [12] $n=405$ & Both & Bowel injury, blood loss, age \\
Okabayashi [13] $n=152$ & Incisional & BMI, bile leakage, blood loss, glucose control \\
Harimoto [7] $n=125$ & Both & Blood loss \\
Arikawa [26] $n=171$ & Organ/space & Blood loss, bile leakage \\
Elola-Olaso [8] $n=2,332$ & Both or organ/space & Operative duration, smoking, dialysis, serum sodium, albumin, bilirubin, open wound \\
Sadamori [3] $n=359$ & Organ/space & Repeat hepatectomy, bile leakage \\
Nakahira [27] $n=911$ & Both & Drain placement, operative duration \\
Kurmann [28] $n=116$ & Both & Hepatic steatosis \\
Scilletta [29] $n=181$ & Both & Operative duration \\
This study $n=226$ & Incisional Organ/space & Anemia, ASA, liver disease, operative duration Concomitant bowel surgery \\
\hline
\end{tabular}

$B M I$ body mass index, ASA American society of anesthesiologists

operation time, simultaneous resection of the liver and colon should be avoided.

We reported the differing risk factors for incisional and organ/space SSIs after liver resection. The relatively small number of incisional SSIs $(n=29)$ and organ/space $(n=9)$ SSIs patients is a limitation in our study. Especially in regard to the patients with organ/space SSIs, the number was quite small. This may be the reason why concomitant bowel and liver surgery was identified as the only risk factor identified for organ/space SSIs. These results should be confirmed in a larger cohort of patients in the future study. This article alarms the clinical importance and difference of incisional SSIs and organ/space SSIs.

In conclusion, risk factors for incisional SSIs included a preoperative compromised condition and prolonged operation times. In contrast, only concomitant bowel surgery was a risk factor for organ/space SSIs. Precautions specifically designed for avoiding organ/space and incisional SSIs may prevent each type of SSI, and thus, may shorten the hospital stay.

Conflict of interest No financial disclosure or competing interests.

\section{References}

1. Imamura H, Seyama Y, Kokudo N et al (2003) One thousand fifty-six hepatectomies without mortality in 8 years. Arch Surg 138:1198-1206

2. Fan ST, Lo CM, Liu CL et al (1999) Hepatectomy for hepatocellular carcinoma: toward zero hospital deaths. Ann Surg 229:322-330

3. Sadamori H, Yagi T, Shinoura S et al (2013) Risk factors for major morbidity after liver resection for hepatocellular carcinoma. Br J Surg 100:122-129

4. Virani S, Michaelson JS, Hutter MM et al (2007) Morbidity and mortality after liver resection: results of the patient safety in surgery study. J Am Coll Surg 204:1284-1292
5. Poon RT, Fan ST, Lo CM et al (2004) Improving perioperative outcome expands the role of hepatectomy in management of benign and malignant hepatobiliary diseases: analysis of 1222 consecutive patients from a prospective database. Ann Surg 240:698-708

6. Cheung TT, Poon RT, Yuen WK et al (2013) Long-term survival analysis of pure laparoscopic versus open hepatectomy for hepatocellular carcinoma in patients with cirrhosis: a singlecenter experience. Ann Surg 257:506-511

7. Harimoto N, Shirabe K, Abe T et al (2011) Prospective randomized controlled trial investigating the type of sutures used during hepatectomy. World J Gastroenterol 17:2338-2342

8. Moreno Elola-Olaso A, Davenport DL, Hundley JC et al (2012) Predictors of surgical site infection after liver resection: a multicentre analysis using National Surgical Quality Improvement Program data. HPB 14:136-141

9. Hübner M, Diana M, Zanetti G et al (2011) Surgical site infections in colon surgery: the patient, the procedure, the hospital, and the surgeon. Arch Surg 146:1240-1245

10. Romy S, Eisenring MC, Bettschart V et al (2008) Laparoscope use and surgical site infections in digestive surgery. Ann Surg 247:627-632

11. Ho VP, Stein SL, Trencheva K et al (2011) Differing risk factors for incisional and organ/space surgical site infections following abdominal colorectal surgery. Dis Colon Rectum 54:818-825

12. Kobayashi S, Gotohda N, Nakagohri T et al (2009) Risk factors of surgical site infection after hepatectomy for liver cancers. World J Surg 33:312-317

13. Okabayashi T, Nishimori I, Yamashita K et al (2009) Risk factors and predictors for surgical site infection after hepatic resection. J Hosp Infect 73:47-53

14. Tsujita E, Yamashita Y, Takeishi K et al (2012) Subcuticular absorbable suture with subcutaneous drainage system prevents incisional SSI after hepatectomy for hepatocellular carcinoma. World J Surg 36:1651-1656

15. Seyama Y, Kokudo N (2009) Assessment of liver function for safe hepatic resection. Hepatol Res 39:107-116

16. Bray GA (1987) Overweight is risking fate. Defenition, classification, prevalence, and risks. Ann N Y Acad Sci 499:14-28

17. Mangram AJ, Horan TC, Pearson ML et al (1999) Guideline for prevention of surgical site infection, 1999. Hospital Infection Control Practices Advisory Committee. Infect Control Hosp Epidemiol 20:250-278 
18. Horan TC, Gaynes RP, Martone WJ et al (1992) CDC definitions of nosocomial surgical site infections, 1992: a modification of CDC definitions of surgical wound infections. Infect Control Hosp Epidemiol 13:606-608

19. Bratzler DW, Houck PM (2004) Antimicrobial prophylaxis for surgery: an advisory statement from the National Surgical Infection Prevention Project. Clin Infect Dis 38:1706-1715

20. Sun HC, Qin LX, Lu L et al (2006) Randomized clinical trial of the effects of abdominal drainage after elective hepatectomy using the crushing clamp method. Br J Surg 93:422-426

21. Dindo D, Demartines N, Clavien PA (2004) Classification of surgical complications: a new proposal with evaluation in a cohort of 6336 patients and results of a survey. Ann Surg 240:205-213

22. Lawson EH, Hall BL, Ko CY (2013) Risk factors for superficial vs deep/organ-space surgical site infections: implications for quality improvement initiatives. JAMA Surg 148:849-858

23. Astagneau P, Rioux C, Golliot F et al (2001) Morbidity and mortality associated with surgical site infections: results from the 1997-1999 INCISO surveillance. J Hosp Infect 48:267-274

24. Yoshimura Y, Kubo S, Hirohashi K et al (2003) Plastic iodophor drape during liver surgery operative use of the iodophorimpregnated adhesive drape to prevent wound infection during high risk surgery. World J Surg 27:685-688
25. Togo S, Kubota T, Takahashi $\mathrm{T}$ et al (2008) Usefulness of absorbable sutures in preventing surgical site infection in hepatectomy. J Gastrointest Surg 12:1041-1046

26. Arikawa T, Kurokawa T, Ohwa Y et al (2011) Risk factors for surgical site infection after hepatectomy for hepatocellular carcinoma. Hepatogastroenterology 58:143-146

27. Nakahira S, Shimizu J, Miyamoto A et al (2013) Proposal for a sub-classification of hepato-biliary-pancreatic operations for surgical site infection surveillance following assessment of results of prospective multicenter data. J Hepatobiliary Pancreat Sci 20:504-511

28. Kurmann A, Wanner B, Martens F et al (2014) Hepatic steatosis is associated with surgical-site infection after hepatic and colorectal surgery. Surgery 156:109-116

29. Scilletta R, Pagano D, Spada M et al (2014) Comparative analysis of the incidence of surgical site infections in patients with liver resection for colorectal hepatic metastases after neoadjuvant chemotherapy. J Surg Res 118:183-189

30. Kirby JB, Liang L, Chen HJ et al (2012) Race, place, and obesity: the complex relationships among community racial/ethnic composition, individual race/ethnicity, and obesity in the United States. Am J Public Health 102:1572-1578

31. Fahy BN, Fischer CP (2012) Synchronous resection of colorectal primary and hepatic metastasis. J Gastrointest Oncol 3:48-58 